University of Massachusetts Amherst

ScholarWorks@UMass Amherst

Chemistry Department Faculty Publication Series

Chemistry

1988

\title{
Network Flow Injection Manifolds for Sample Dilution and Calibration in Flame Atomic Absorption Spectrometry
}

Julian Tyson

University of Massachusetts Amherst

S. R. Bysouth

Loughborough University of Technology

Follow this and additional works at: https://scholarworks.umass.edu/chem_faculty_pubs

Part of the Analytical Chemistry Commons

\section{Recommended Citation}

Tyson, Julian and Bysouth, S. R., "Network Flow Injection Manifolds for Sample Dilution and Calibration in Flame Atomic Absorption Spectrometry" (1988). Journal of Analytical Atomic Spectrometry. 1401.

Retrieved from https://scholarworks.umass.edu/chem_faculty_pubs/1401 


\title{
Network Flow Injection Manifolds for Sample Dilution and Calibration in Flame Atomic Absorption Spectrometry*
}

\author{
Julian F. Tyson and Stephen R. Bysouth \\ Department of Chemistry, University of Technology, Loughborough, Leicestershire LE11 3TU, UK
}

\begin{abstract}
Two flow injection manifolds for on-line dilution are described and evaluated. One consists of an asymmetrical two-branch network giving rise to three measurement points, namely the two maxima and the minimum between the partially overlapping peaks. With this manifold a concentration range for magnesium of $0.2-100 \mathrm{mg} \mathrm{I}^{-1}$ was covered with a relative standard deviation of about $2 \%$. The relative dilutions at the measurement points were 1.0, 5.9 and 23, corresponding to absolute values of 3.4, 20 and 77, respectively. The other manifold consisted of a three-branch network giving three partially overlapping peaks and five measurement points, three maxima and two minima; dilution factors of $6.8,10,14,22$ and 28 were produced. A calibration generated by the injection of ${ }^{6} \mathrm{mg} \mathrm{I}^{-1}$ magnesium solution was used to analyse solutions in the range $0.2-10 \mathrm{mg} \mathrm{l}^{-1}$. The corresponding range of standard deviations was $0.017-0.30 \mathrm{mg}^{-1}$. This method of calibration is only possible if straight line calibration graphs are obtained. The method generates a family of calibration graphs of varying sensitivity covering the extended concentration range that are more readily visualised on log-log plots. For dilution of off-range samples and extending the conventional calibration range, the two-branch network is preferred as it is less susceptible to minor fluctuations in flow-rate in those branches of the manifold which gave rise to poor day to day reproducibility of the three-branch network.
\end{abstract}

Keywords: Flow injection; network manifolds; on-line dilution; extended range calibration and single standard calibration; flame atomic absorption spectrometry

Flow injection (FI) techniques have been used for a wide variety of pre-treatment procedures ${ }^{1,2}$ for atomic spectrometry. In addition to chemical manipulations such as matrix modification (by ion exchange, solvent extraction or vapour generation), several methods of exploiting the controlled dispersion characteristics of FI manifolds for on-line dilution of samples and standards have been described. These methods include the use of merging streams ${ }^{3}$ and zones, ${ }^{4}$ control of sample volume, ${ }^{5}$ variation of manifold dimensions ${ }^{6}$ and a number of time-based methods such as zone sampling, ${ }^{7}$ peak-width measurement ${ }^{8}$ and continuous dilution. ${ }^{9}$ The recent literature concerning FI techniques for atomic spectrometry, including flame atomic absorption spectrometry (FAAS), has been reviewed. ${ }^{10}$ Although it is not necessarily so, most FI manifolds used in conjunction with FAAS result in decreased sensitivity when peak height absorbance, the most commonly used analytical parameter, is compared with the steady-state absorbance value. Thus FI procedures contain an inherent dilution step. The manifolds that have been specifically designed to produce substantial dilution of the injected solution can be divided into two types: those that have a fixed dilution factor and those that have a variable dilution factor. This latter category of manifold can be further sub-divided into those manifolds capable of producing continuously variable dilution ${ }^{5,7,9}$ and those that are capable of several discrete dilution stages. ${ }^{6}$ With the first type of manifold calibration is performed in the usual way. A set of calibration standards is prepared in calibrated glassware and introduced sequentially via the FI manifold. With the second type of manifold, calibration is achieved with a single standard solution as the working range is covered by sequential selection of a variety of dilution factors by alteration of the appropriate manifold parameter.

In this paper a method of achieving a variety of dilution factors for a single injection without the need for controlled timing of any operation is described. The manifold used is

\footnotetext{
* This paper is dedicated to the memory of Professor John M
} Ottaway referred to as a "network," i.e., it consists of, in general, several branching and confluence points. The manifolds used here contained one of each type of point. If the network consists of tubing of different dimensions then the injected zone will be unequally split between the branches, and as residence times of the sub-sampled zones in each branch will differ, on recombination multiple peaks will be produced. In general, for $n$ unequal branches, $n$ peaks will be produced. The simplest network manifold is shown in Fig. 1(a) and the resulting instrument response in Fig. 1(b). The generation of multiple peaks is normally used in flow injection techniques for the "simultaneous" determination of several analytes in the same sample. ${ }^{11}$ However, one of the earliest publications on $\mathrm{FI}^{12}$ described the manifold shown in Fig. 1(a) for the determination, by solution spectrophotometry, of chloride. Peak ratios of up to $10: 1$ were shown to be possible by appropriate choice of dimensions for the tubing between the split and confluence points, though for the analysis of real samples (river, estuary and sea water) a $3: 1$ ratio was used. The same manifold has been studied in detail by Fernandez et al. 13 who established experimentally the relationship between the ratio of the peak heights (expressed as a ratio of dispersion coefficients $\dagger$ ) and a number of experimental variables including tube length, flow-rate, volume injected and temperature. A network manifold containing ionexchange mini-columns has been devised for the simultaneous determination of chloride and nitrate. ${ }^{14}$

In all of these previously described uses of network manifolds, the analytical parameter used was maximum peak height. In the work described here, use is made of both the instrument response at the minimum between peaks and the response at the peak maximum.

† Dispersion coefficient is defined as the ratio of the injected concentration to that at the point of measurement (usually peak maximum). For an instrument with a linear response, the dispersion coefficient may be found from the ratio of steady-state response to that at the point of measurement. 

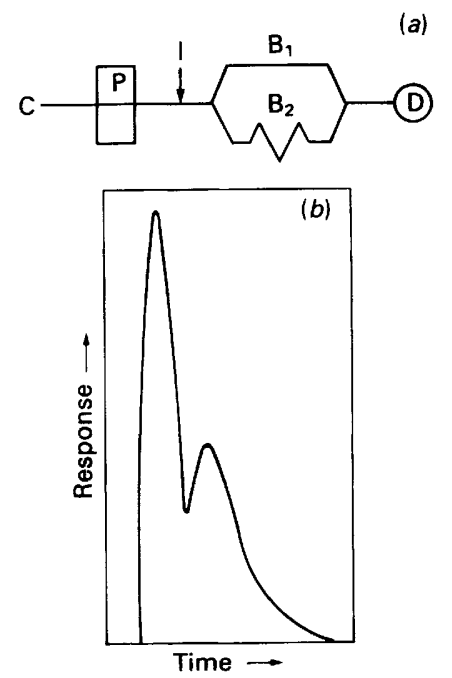

Fig. 1. (a) Two-branch network manifold for FI-AAS: C, carrier stream; $P$, peristaltic pump; $I$, injection valve; $B_{1}$ and $B_{2}$, branches of network; $\mathrm{D}$, detector. (b) Schematic instrument response for single injection at I

\section{Experimental}

\section{Apparatus}

The flow injection manifold consisted of a P.S. Analytical T-series rotary injection valve, an LKB Microperpex peristaltic pump and $0.65 \mathrm{~mm}$ internal diameter PTFE tubing. For the two-branch network, the split and confluence connectors were $Y$-pieces constructed in-house, for the three-branch manifold Alltech type 05-40-5108 connectors were used. These are similar to the AutoAnalyser PT10 connectors. The connectors were mounted vertically to avoid the accumulation of small air bubbles at the branch points. The atomic absorption spectrometer was a Baird Atomic Model A3400 and the absorbance - time response was recorded on a Philips Model AR55 chart recorder. Magnesium was used as the test element and the instrument was optimised for maximum sensitivity in the usual manner.

\section{Procedure}

\section{Two-branch network}

The manifold parameters [see Fig. 1(a)] were: carrier, distilled water at a flow-rate of $6 \mathrm{ml} \mathrm{min}^{-1}$; volume injected, $85 \mu \mathrm{l}$; length of branch $B_{1}, 173 \mathrm{~mm}$; length of branch $B_{2}, 600 \mathrm{~mm}$. Between the injection valve and the stream-split point there was $110 \mathrm{~mm}$ of tubing, and between the confluence point and detector, $240 \mathrm{~mm}$ of tubing. Solutions covering the range $0.1-100 \mathrm{mg} \mathrm{I}^{-1}$ of $\mathrm{Mg}$ were injected sequentially and the resultant multiple peak recorded. The dispersion coefficient corresponding to the two maxima and the minimum were calculated. Ten replicate injections of solutions containing $1.5,15$ and $40 \mathrm{mg} \mathrm{l}^{-1}$ of $\mathrm{Mg}$ were made.

\section{Three-branch network}

The manifold is shown schematically in Fig. 2(a). The detailed arrangement of the vertically mounted connectors and connecting tubing between the split and confluence "points" is shown in Fig. 2(b). The carrier stream was water flowing at $6 \mathrm{ml} \mathrm{min}^{-1}$, the volume injected was $82 \mu \mathrm{l}$ and the lengths of branches $B_{1}, B_{2}$ and $B_{3}$ were 237,433 and $635 \mathrm{~mm}$, respectively. Between the injection valve and stream-split point were $112 \mathrm{~mm}$ of tubing, and between the confluence point and detector $152 \mathrm{~mm}$ of tubing. A series of solutions covering the range $0.2-20 \mathrm{mgl}^{-1}$ of $\mathrm{Mg}$ were injected. Six replicate injections of each solution were made. The short-term (a)

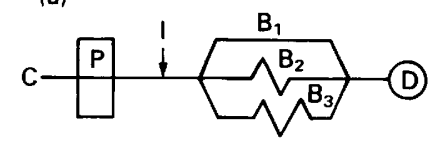

(b)

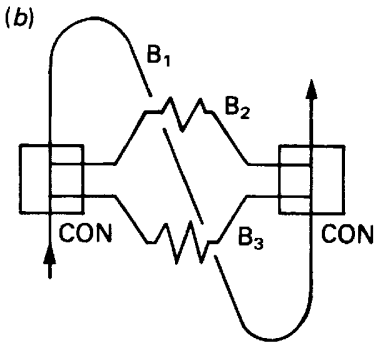

Fig. 2. (a) Three-branch network manifold: C, carrier stream; P, peristaltic pump; $I$, injection valve; $B_{1}, B_{2}$ and $B_{3}$, branches of network; D, detector. (b) Arrangement of split and confluence connectors (CON)

Table 1. Results for the two-branch network

\begin{tabular}{cccc} 
& \multicolumn{3}{c}{ Absorbance } \\
Concentration \\
injected/mg l ${ }^{-1}$ & Peak 1 & Minimum & Peak 2 \\
0.10 & 0.030 & - & - \\
0.30 & 0.088 & - & 0.008 \\
0.50 & 0.152 & 0.004 & 0.024 \\
0.70 & 0.220 & 0.006 & 0.038 \\
1.00 & 0.306 & 0.008 & 0.052 \\
10.0 & - & 0.136 & 0.484 \\
20 & - & 0.254 & 0.900 \\
30 & - & 0.400 & 1.264 \\
40 & - & 0.504 & - \\
50 & - & 0.644 & - \\
100 & - & 1.208 & - \\
\hline
\end{tabular}

precision of the manifold was evaluated by the replicate injection of a $2 \mathrm{mgl}^{-1} \mathrm{Mg}$ solution. The experiment was repeated the following day.

\section{Results and Discussion}

\section{Two-branch Network}

The values of the peaks and minimum obtained with the two-branch network are given in Table 1 . The absence of an entry in a particular column indicates that the reading was off-scale (either below the detection limit or above the response range of the instrument). The relative standard deviations of the $1.5,15$ and $40 \mathrm{mg} \mathrm{l}^{-1}$ solutions were $1.3,1.4$ and $2.6 \%$, respectively. The relative dilutions obtained at peak 1 , peak 2 and the minimum were $1.0,5.9$ and 23 , corresponding to dispersion coefficients of approximately 3.4 , 20 and 77 , respectively. To illustrate the high dilution capacity of this manifold, five replicate injections of the $100 \mathrm{mgl}^{-1}$ solution are shown in Fig. 3, illustrating the use of the minimum between the peaks as a measurement point when the peak maxima are "off scale." 9 The results show that a manifold of this type allows the extension of the working range up to $100 \mathrm{mgl}^{-1}$, which is approximately two orders of magnitude longer than the conventional working range. As the dilution produced is at least a factor of 3.4 (peak 1 ), it would be expected that the lower limit of the useful working range of the network manifold would be about this factor higher than the conventional lower limit.

\section{Three-branch Network}

Six replicate injections were made of a $2 \mathrm{mgl}^{-1}$ solution as shown in Fig. 4, and the absorbance concentration data produced are given in Table 2 . Each entry is the mean of six 


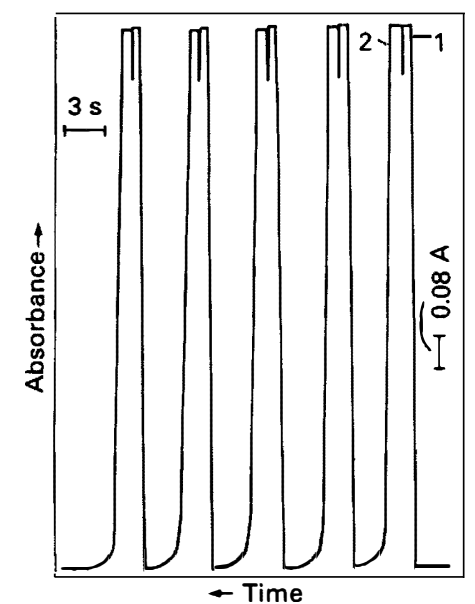

Fig. 3. Chart recordings for replicate injections of a $100 \mathrm{mg} \mathrm{l}^{-1}$ magnesium solution into the two-branch network

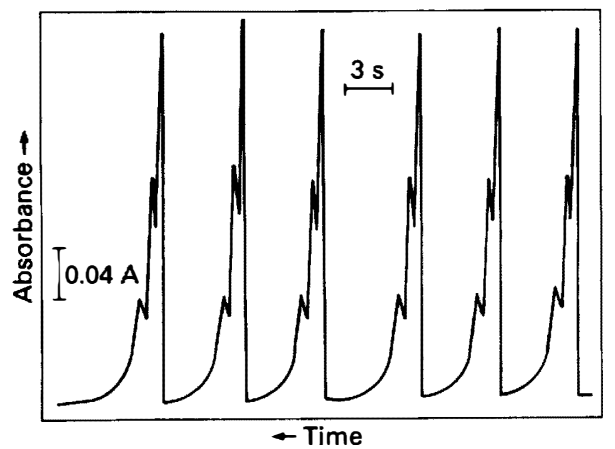

Fig. 4. Chart recordings for replicate injections of a $2 \mathrm{mgl}^{-1}$ magnesium solution into the three-branch network

replicates and the corresponding relative standard deviations are given in Table 3 . These results give rise to five calibration graphs of different sensitivity as shown in Fig. 5. The results of a least-squares regression analysis of the data in Table 2 are given in Table 4, from which it can be seen that the calibration graphs are straight lines. Thus a ratio of instrument responses can be assumed to be equal to a ratio of concentrations and if the steady-state responses of the instruments were known, dispersion coefficient values for the five measurement points could be calculated. However, it is not necessary to know the steady-state response in order to use this manifold to calculate the concentrations of unknowns when it is known that the calibration function is linear. To illustrate this concept the injections of the $6 \mathrm{mg} \mathrm{l}^{-1}$ solution may be used to calibrate the system for the analysis of the other solutions as unknowns. The basis of the method is as follows: let the steady-state absorbance for $6 \mathrm{mg} \mathrm{l}^{-1}$ be $s$ (a hypothetical value on an infinitely long linear calibration range), thus if the responses at the measurement points are $p_{1}, m_{1}, p_{2}, m_{2}, p_{3}$ (where $p$ indicates a peak absorbance and $m$ a minimum absorbance), then the dispersion coefficients of the five points are $s / p_{1}, s / m_{1}$, $s / p_{2}, s / m_{2}$ and $s / p_{3}$, respectively. When an unknown of concentration $x \mathrm{mg} \mathrm{l}^{-1}$ is injected, then five absorbance values are obtained, namely, $p_{x 1}, m_{x 1}, p_{x 2}, m_{x 2}$ and $p_{x 3}$ and the corresponding hypothetical steady-state value would be $s x / 6$. Thus the dispersion coefficients of the five points would be given by $s x / 6 p_{x 1}, s x / 6 m_{x 1}, s x / 6 p_{x 2}, s x / 6 m_{x 2}$ and $s x / 6 p_{x 3}$. Equating these values with the corresponding values calculated on the basis of the $6 \mathrm{mg} \mathrm{l}^{-1}$ solution gives

$$
x=6 p_{x 1} / p_{1}=6 m_{x 1} / m_{1}=6 p_{x 2} / p_{2}=6 m_{x 2} / m_{2}=6 p_{x 3} / p_{3}
$$

The results of this calculation are given in Table 5. Only one result (that for the $0.60 \mathrm{mgl}^{-1}$ solution) shows bias at the $95 \%$ confidence level, although to some extent this is due to the fairly large values of the standard deviations obtained.
Table 2. Results for the three-branch network

\begin{tabular}{cccccc} 
& \multicolumn{5}{c}{ Absorbance } \\
\cline { 2 - 6 } Concentration \\
\cline { 2 - 6 } injected/mg l ${ }^{-1}$ & $p_{1}$ & $m_{1}$ & \multicolumn{1}{c}{$p_{2}$} & \multicolumn{1}{c}{$m_{2}$} & \multicolumn{1}{c}{$p_{3}$} \\
0.00 & 0.000 & 0.000 & 0.000 & 0.000 & 0.000 \\
0.20 & 0.0273 & 0.015 & 0.019 & 0.0083 & 0.011 \\
0.40 & 0.0604 & 0.0291 & 0.0372 & 0.0141 & 0.0188 \\
0.60 & 0.086 & 0.0384 & 0.050 & 0.0183 & 0.0267 \\
1.0 & 0.154 & 0.0671 & 0.0899 & 0.0321 & 0.0444 \\
2.0 & 0.307 & 0.140 & 0.186 & 0.0674 & 0.0899 \\
4.0 & 0.605 & 0.303 & 0.387 & 0.147 & 0.193 \\
6.0 & 0.863 & 0.445 & 0.586 & 0.222 & 0.292 \\
10.0 & - & 0.726 & 0.934 & 0.376 & 0.494 \\
20.0 & - & - & - & 0.693 & 0.915 \\
\hline
\end{tabular}

Table 3. Short-term precision of the three-branch network

\begin{tabular}{cccccc} 
& \multicolumn{5}{c}{ Relative standard deviation $(n=6), \%$} \\
\cline { 2 - 6 } Concentration \\
injected $/ \mathrm{mg} \mathrm{I}^{-1}$ & $p_{1}$ & $m_{1}$ & $p_{2}$ & $m_{2}$ & $p_{3}$ \\
0.2 & 3.9 & 6.9 & 2.8 & 11 & 7.9 \\
0.4 & 0.84 & 2.2 & 3.0 & 5.5 & 3.3 \\
0.6 & 1.1 & 5.2 & 3.2 & 5.0 & 4.1 \\
1.0 & 1.6 & 4.5 & 1.2 & 3.2 & 3.2 \\
2.0 & 1.6 & 6.3 & 3.1 & 3.5 & 1.2 \\
4.0 & 1.1 & 1.7 & 1.0 & 0.7 & 0.9 \\
6.0 & 1.5 & 0.56 & 0.78 & 1.2 & 1.2 \\
10.0 & - & 1.8 & 1.0 & 1.2 & 1.2 \\
20.0 & - & - & - & 1.5 & 0.63 \\
\hline
\end{tabular}

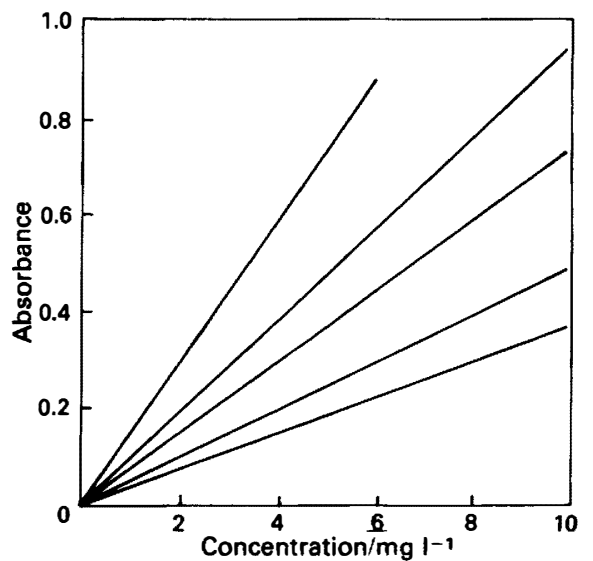

Fig. 5. Family of calibration graphs for the three-branch network

In general with atomic absorption instruments, calibrations are not linear and thus this approach will be correspondingly less accurate as curvature increases. The manifold allows an increase in the working range of the instrument and for magnesium this would be to about $30 \mathrm{mg} \mathrm{l}^{-1}$ as the dispersion coefficients of the measurement points are approximately 6.8 , $10,14,22$ and 28 for peak 1 , peak 2 , minimum 1 , peak 3 and minimum 2 , respectively. As this range is too long to be usefully plotted on linear co-ordinates, the effect of transforming to $\log -\log$ co-ordinates is shown in Fig. 6.

The day to day variation in relative absorbance values is given in Table 6 . The changes observed may all be explained on the basis of a decrease in the flow in $\mathrm{B}_{1}$ [see Fig. 2(a)]. This will increase the flow in the other two branches and thus the volume of the sub-sampled zones. This accounts for an increase in peaks 2 and 3 compared with peak 1 . Thus, compared with the day 1 situation, the sample in $B_{1}$ is moving more slowly and the sample in $\mathrm{B}_{2}$ more quickly and thus the peaks will be closer together and the minimum between them will increase (as is seen in Table 6). It can be seen from the change in heights of peaks 2 and 3 that the fluid in $B_{2}$ is flowing 
Table 4. Least-squares regression analysis of calibration data from the three-branch network

\begin{tabular}{|c|c|c|c|c|c|c|}
\hline Data point & & $\begin{array}{c}\text { Slope/ } \\
\mathrm{A} \mathrm{mg}^{-1} \mathrm{l}\end{array}$ & $\begin{array}{c}\text { Intercept/ } \\
\mathrm{A}\end{array}$ & $\begin{array}{c}\text { Correlation } \\
\text { coefficient }\end{array}$ & $\begin{array}{c}\text { Standard } \\
\text { deviation of } \\
\text { residual/A }\end{array}$ & $\begin{array}{l}\text { Wald - } \\
\text { Wolfowitz } \\
\text { runs test* }\end{array}$ \\
\hline Peak $1 \quad \ldots$. & .. & 0.146 & 0.004 & 0.9994 & 0.012 & $-\dagger$ \\
\hline Minimum 1 & .. & 0.073 & -0.001 & 0.9997 & 0.006 & $-\dagger$ \\
\hline Peak 2 & $\ldots$ & 0.095 & -0.0003 & 0.9996 & 0.010 & NS \\
\hline Minimum $2 \ldots$ & $\ldots$ & 0.038 & -0.003 & 0.9997 & 0.003 & NS \\
\hline Peak $3 \quad$. & $\ldots$ & 0.049 & -0.003 & 0.9998 & 0.003 & NS \\
\hline
\end{tabular}

${ }^{*}$ This test ${ }^{15}$ examines the sequences of positive and negative residuals obtained for a non-random over-all sequence; NS indicates the test showed a random over-all sequence, i.e., no evidence of curvature from the signs of the residuals.

$\dagger$ The sequence of positive and negative values fell outside the scope of the test.

Table 5. Single-standard calibration with the three-branch manifold

Unknown
concentration/
mg l-1
0.20
0.40
0.60
1.00
2.00
4.00
10.00

\begin{tabular}{cccccc}
\multicolumn{6}{c}{ Concentration found $/ \mathrm{mg} \mathrm{l}^{-1}$} \\
\hline$p_{1}$ & $m_{1}$ & $p_{2}$ & $m_{2}$ & $p_{3}$ & Mean \\
0.190 & 0.202 & 0.194 & 0.224 & 0.226 & 0.207 \\
0.420 & 0.393 & 0.379 & 0.381 & 0.385 & 0.392 \\
0.597 & 0.518 & 0.510 & 0.494 & 0.547 & 0.533 \\
1.07 & 0.906 & 0.917 & 0.867 & 0.910 & 0.934 \\
2.13 & 1.89 & 1.90 & 1.82 & 1.84 & 1.92 \\
4.20 & 4.09 & 3.95 & 3.97 & 3.96 & 4.03 \\
- & 9.80 & 9.53 & 10.2 & 10.1 & 9.91
\end{tabular}

$\begin{gathered}\text { Standard } \\ \text { deviation/ } \\ \mathrm{mg} \mathrm{l}^{-1}\end{gathered}$
0.017
0.018
0.041
0.078
0.12
0.11
0.30

$95 \%$

Confidence

interval

0.021

0.021

0.050

0.098

0.15

0.14

0.38

Table 6. Precision of the three-branch network

\begin{tabular}{|c|c|c|c|c|c|c|c|}
\hline Parameter & & & Peak 1 & Peak 2 & Minimum 1 & Peak 3 & Minimum 2 \\
\hline \multicolumn{8}{|l|}{ Day $1-$} \\
\hline Mean relative peak height $\ldots$ & . . & . & 1 & 0.52 & 0.39 & 0.25 & 0.19 \\
\hline Relative standard deviation, $\%$ & . & . & 0 & 3.8 & 2.3 & 2.8 & 6.1 \\
\hline \multicolumn{8}{|l|}{ Day 2- } \\
\hline Mean relative peak height $\ldots$ & $\cdots$ & $\ldots$ & 1 & 0.58 & 0.45 & 0.26 & 0.18 \\
\hline Relative standard deviation, $\%$ & . & . & 0 & 2.0 & 1.9 & 9.7 & 2.7 \\
\hline
\end{tabular}

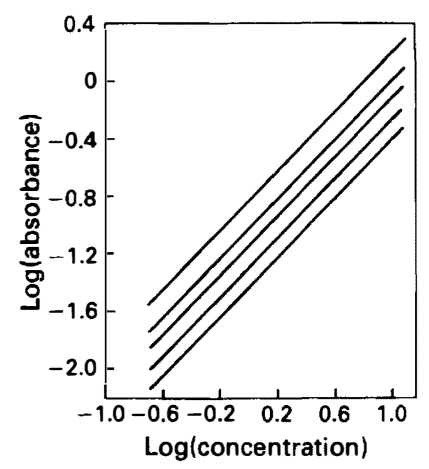

Fig. 6. Log - log calibration plots for the three-branch network

faster than the fluid in $\mathrm{B}_{3}$ compared with the day 1 situation and thus the peaks will be further apart and the minimum between them will decrease (as is shown in Table 6).

The short-term precision is poorer than would normally be expected for FI-FAAS in which a single peak was obtained. Values for relative standard deviations of about $2 \%$ might typically be obtained at absorbance values well above the detection limit. It was observed that the peak heights (and minimum) were more affected by the presence of small air bubbles in the manifold than would be so for a single-line manifold. Although efforts were made to keep the manifold free from accumulated air bubbles, it is difficult to keep manifolds entirely free. De-gassing of solutions is of little value as PTFE tubing is porous to gases, and solutions rapidly take up gases during residence in the manifold. The tubing and connectors are not completely transparent and visual inspection of a manifold may not reveal the presence of a small air bubble which will restrict the flow in that branch of the manifold. Particulate material, such as filter-paper fibres, may also cause partial blockage of manifold tubing and could thus give rise to similar problems. It is possible that manifolds of this type, which are primarily designed to produce large dilution factors, could be constructed of tubing of wider bore than the usual $0.5-0.8 \mathrm{~mm}$ internal diameter tubing.

\section{Conclusions}

Large dilution factors may be obtained by using the minimum between two overlapping peaks generated from an asymmetric two-branch network manifold. In conjunction with the appropriate peak values, the calibration range can be extended by at least two orders of magnitude with a relatively small sacrifice in detection limit. As with other FI methods of extending the working range upwards, the method may be used for analyses in situations where the best precision obtainable from FAAS is not required. Alternatively the method provides a rapid means of calculating a dilution factor to be used in conjunction with a more precise calibration strategy.

Attempts to obtain calibration data from a single injection into a three-branch network manifold were only partially successful. Although five calibration points can be obtained from a single injection, the dispersion coefficients associated with each of these points cannot be determined with any certainty and thus the concentrations corresponding to each point cannot be accurately calculated. However, the shortterm variations in dilution factors are sufficiently small for the manifold to be used as an on-line dilution device, with calibration by the injection of standards prepared by serial dilution in the normal way. However, in this mode of operation, the three-branch network offers nothing by way of advantage over the two-branch network and the latter design would be preferred, as it is less susceptible to fluctuations in the flow-rates in the branches of the network. If the drift in the 
dispersion coefficients for the three-branch manifold could be reduced, a manifold of this type covering a wider range of dilution values than the manifold described here could have potential as a practical dilution and calibration accessory for FAAS.

The authors thank the Trustees of the Analytical Chemistry Trust Fund of the Royal Society of Chemistry for the award of an SAC Research studentship.

\section{References}

1. Tyson, J. F., Analyst, 1985, 110, 419

2. Růžička, J., Fresenius Z. Anal. Chem., 1986, 324, 745.

3. Alonso, J., Bartroli, J., Lima, J. L. F. C., and Machado, A. A. S. C., Anal. Chim. Acta, 1986, 179, 503.

4. Zagatto, E. A. G., Krug, F. J., Bergamin, F. ${ }^{\circ}$, H., Jorgensen, S. S., and Reis, B. F., Anal. Chim. Acta, 1979, 104, 279.

5. Sherwood, R. A., Rocks, B. F., and Riley, C., Analyst, 1985, 110, 493.
6. Tyson, J. F., Mariara, J. R., and Appleton, J. M. H., J. Anal. At. Spectrom., 1986, 1, 273.

7. Reis, B. F., Jacintho, A. O., Mortatti, J., Krug, F. J., Zagatto, E. A. G., Bergamin, F. ${ }^{\circ}$ H., and Persenda, L. C. R., Anal. Chim. Acta, 1981, 123, 221.

8. Tyson, J. F., Analyst, 1984, 109, 319.

9. Tyson, J. F., and Appleton, J. M. H., Talanta, 1984, 31, 9.

10. Marshall, J., Haswell, S. J., and Hill, S. J., J. Anal. At. Spectrom., 1987, 2, 79R.

11. Luque de Castro, M. D., and Valcárcel Cases, M., Analyst 1984, 109, 413.

12. Růžička, J., Stewart, J. W. B., and Zagatto, E. A. G., Anal. Chim. Acta, 1976, 81, 387.

13. Fernandez, A., Gomez-Nieto, M. A., Luque de Castro, M. D. and Valcárcel, M., Anal. Chim. Acta, 1984, 165, 217.

14. Faizullah, A. T., and Townshend, A., Anal. Chim. Acta, 1986, 179, 233.

15. Miller, J. C., and Miller, J. N., "Statistics for Analytical Chemistry," Ellis Horwood, Chichester, 1984, p. 123.

Paper J7/81

Received July 7th, 1987 Accepted August 21st, 1987 